Relations industrielles

Industrial Relations

\title{
Le changement planifié, par Pierre Collerette et Gilles Delisle, Montréal, L’Agence d'Arc Ltée Inc., 1982, 213 pp. ISBN 0-89022-045-1.
}

\section{Laurent Bélanger}

Volume 38, numéro 1, 1983

URI : https://id.erudit.org/iderudit/029347ar

DOI : https://doi.org/10.7202/029347ar

Aller au sommaire du numéro

Éditeur(s)

Département des relations industrielles de l'Université Laval

ISSN

0034-379X (imprimé)

1703-8138 (numérique)

Découvrir la revue

Citer ce compte rendu

Bélanger, L. (1983). Compte rendu de [Le changement planifié, par Pierre Collerette et Gilles Delisle, Montréal, L’Agence d'Arc Ltée Inc., 1982, 213 pp. ISBN 0-89022-045-1.] Relations industrielles / Industrial Relations, 38(1), 195-196. https://doi.org/10.7202/029347ar

Tous droits réservés ㄷ Département des relations industrielles de l'Université Laval, 1983
Ce document est protégé par la loi sur le droit d'auteur. L’utilisation des services d’Érudit (y compris la reproduction) est assujettie à sa politique d'utilisation que vous pouvez consulter en ligne.

https://apropos.erudit.org/fr/usagers/politique-dutilisation/ 
kinds of crimes show a rising trend... The city's growth has miserably failed to accelerate changes in the surrounding region of which it is a part. Urban growth in the region has failed to generate economic growth and cultural change."

Dr. Sahai's empirical study is a pioneering work in the field of urban sociology in the sense that it has brought a fresh perspective on the relationship between urbanization and regional development in a developing country. However, the author does not give the reasons why Duragapur Steel city has failed to rise to the status of 'generative city' and has remained a 'parasitic city'. This could be another fruitful area of research.

\section{Hem C. JAIN}

University of New Brunswick

Le changement planifié, par Pierre Collerette et Gilles Delisle, Montréal, L'Agence d'Arc Ltée Inc., 1982, 213 pp., ISBN 0-89022-045-1

$\mathrm{Au}$ moment où l'on s'intéresse activement à l'amélioration de la qualité de la vie au travail par la mise en oeuvre de changements majeurs au plan de la structure et du climat des organisations de travail, ou encore au plan de la technologie, la publication de cet ouvrage arrive à point. Au lieu de traiter du contenu des changements, c'est-à-dire telle ou telle modification apportée à un système organisationnel, les auteurs, consultants et professeurs, ont cherché plutôt à décrire le processus même du changement, et, plus précisément, du changement planifié qu'ils définissent comme «un effort délibéré de changer une situation dite insatisfaisante, au moyen d'une suite d'actions dont le choix et l'orchestration résultent d'une analyse systématique de la situation en cause» (p. 49).

Après avoir présenté un cadre conceptuel emprunté à la théorie des systèmes, les auteurs traitent de la notion du changement en général, de ses sources et de ses mécanismes, pour présenter par la suite la démarche pro- pre au changement planifié qui se décompose dans ses phases principales, diagnostic de la situation existante, de la planification des actions, d'exécution et d'évaluation. Au cours du processus complet ou au cours de l'une de ses phases, une foule de considérations entrent en ligne de compte qui font l'objet de chapitres particuliers: processus de formation et de changement d'attitudes; forces restrictives et forces motrices (phénomènes de résistance au changement selon le modèle de Levine); approches du changement (empirico-rationnelles, normatives-rééducatives, coercitives, consensuelles, conflictuelles, contre-culturelles); définition des objectifs au cours d'une intervention; stratégies en fonction du pouvoir dont dispose l'agent de changement.

On se saurait trop insister sur l'importance qu'il faut attacher au processus de changement lorsqu'on réalise rapidement que le succès ou l'échec d'une intervention dans les systèmes sociaux en découle justement.

Il se suffit pas de croire que, par un décret ou par des modifications de politiques, de directives ou de procédures, le changement va se produire automatiquement; il faut laisser le temps aux gens impliqués de réfléchir, de cheminer à leur rythme, vu la situation nouvelle que l'on veut créer: c'est pourquoi le choix d'une démarche, d'une approche ou d'une stratégie devient importante au cours d'une intervention dans les organisations de travail.

Les auteurs n'optent pas pour une approche de changement planifiée plutôt qu'une autre. Ils prennent donc soin de faire les quelques distinctions qui s'imposent entre le changement planifié et le courant du «développement des organisations» d'origine nordaméricaine. Ce dernier s'inspire de valeurs d'autonomie de la personne humaine, de confiance mutuelle, de participation, de développement personnel. La relation d'aide qui se crée et se développe entre l'agent du changement et le système destinataire dans un processus de développement organisationnel diffère généralement du type de relations qui 
s'installent entre l'agent de changement et les destinataires dans une démarche de changement planifié, sauf lorsque le changement emprunte une approche normative- rééducative ou consensuelle. Dans ces deux cas, les types de relations agent de changement et destinataires se rejoignent. Malheureusement, les auteurs ne sont pas très explicites sur ce point et, par conséquent, n'aident pas le consultant en D.O. à se situer à l'intérieur de la démarche du changement planifié.

L'ouvrage est écrit dans un langage accessible à tous, évitant le jargon de l'une ou l'autre des disciplines en sciences sociales et en administration. Il intéressera les cadres hiérarchiques aussi bien que les cadres fonctionnels, plus particulièrement, ceux qui oeuvrent au sein des directions du personnel, ceux qui agissent comme conseillers auprès des organisations de travail et des organismes communautaires. De plus, il m'apparaît indispensable à ceux qui s'intéressent à l'introduction de nouvelles formes d'organisation du travail.

\section{Laurent BÉLANGER}

Université Laval

\section{THE JOURNAL OF INDUSTRIAL RELATIONS}

The Journal of the Industrial Relations Society of Australia

Editor: Professor John Niland, University of New South Wales

Vol. 24, No. 3, September 1982.

Articles: Economics and Industrial Relations, J.E. ISAAC; General Limitations on the 'Right to Fire' in New Zealand, A.J. GEARE; The Influence of Labour Market Fluctuations on School Participation, G. Brian THOMPSON; The Importance of the Interpersonal Relationship between First-Line Supervisor and Subordinate, Robert R. REEDER; New Technology, the Economy and the Unions in Britain, Ray MARKEY. Notes: Worker Involvement - An Analysis of the SECV Working Parties - A Comment, Julian TEICHER; Worker Involvement: An Analysis of the SECV Working Parties - A Reply, John BENSON. Book Reviews. Book Notes.

Published quarterly. Subscription: Australia \$A29, overseas \$A35 (surface mail). Single issues: \$A8.25 plus postage. Order from G.J. Bennett Associates, GPO Box 2260, Sydney, NSW 2001, Australia. 